\title{
Screening for inhibitor development and it's risk factors in patients with severe haemophilia a in Oman
}

\begin{abstract}
Introduction: A major challenging complication of factor VIII replacement therapy is the development of neutralizing antibodies, rendering therapy ineffective. There are recognized genetic and non-genetic risk factors for inhibitors development. This study aimed to screen for the prevalence of inhibitor development among Omani patients with severe haemophilia A and to define its non-genetic risk factors that might help to develop prevention protocols.

Methods: A Retrospective cohort study that included all patients with haemophilia A registered in Oman. Data were collected using computerized hospital information system. Patients' demographic data: MRN, age at diagnosis, age at first treatment, date of the first documented inhibitor development, \& data on non-genetic risk factors such as, mode of treatment (on demand versus prophylaxis), type and dose of the concentrate, time interval of exposure to factor VIII till inhibitor development, previous surgeries or major bleeding events/blood transfusion; were included. Missing data were collected through phone interviews whenever possible.

Results: Out of the 156 patients registered in Oman, 78 patients had complete data. The age of inhibitor development ranged between 16 months and 21years $(109.4+79.9$ months). The prevalence of inhibitors among our patients was $35 \%$ and mainly of low titer $87 \%$. There was a significant correlation between the intensity of prophylaxis and inhibitor development $(\mathrm{p}<0.05)$. Inhibitor development was not associated with type of the factor used, time interval of exposure to factor VIII, use of on-demand or prophylaxis regimen and history of major bleed or previous surgery.

Discussion/Conclusion: Inhibitors development is a major complication in patients with hemophilia A in Oman. A larger scale study that includes all the registered patients and their genetic mutation is needed.
\end{abstract}

Keywords: severe hemophilia A, inhibitors, risk factors, QoL, hemarthrosis, PUP, HIS, SPSS
Volume 6 Issue 3 - 2018

\author{
Hanan Fawzy Nazir, ${ }^{1,2}$ Laila Al khanbashi, ${ }^{3}$ \\ Yasser Ahmed Wali, Alyaa Al Mughairy, \\ Adawiya Al Jamie, ${ }^{3}$ Fatma Al Badi ${ }^{3}$ \\ 'Department of Child Health, Sultan Qaboos University \\ Hospital, Oman \\ ${ }^{2}$ Department of Pediatrics, Alexandria University, Egypt \\ ${ }^{3}$ Department of Child Health, Oman Medical Speciality Board \\ (OMSB), Oman \\ ${ }^{4}$ Department of pediatrics Hematology Oncology, Royal \\ Hospital , Oman
}

\begin{abstract}
Correspondence: Hanan Fawzy Nazir, POB 35, Alkhodh, Zip Code 123, Department of Pediatrics, Alexandria Faculty of Medicine, Child Health Department, Sultan Qaboos University Hospital, Muscat, Oman, Tel 0096896556198 , Fax 00968 24141|36,Email dr.hanannazir@yahoo.com
\end{abstract}

Received: March 27, 2018| Published: May 16, 2018

\section{Introduction}

Hemophilia A is an X-linked recessive bleeding disorder. It is the second most common inherited bleeding disorder with an estimated incidence ranging from 1 in 20,000 to as high as 1 in 10,000 persons. The underlying abnormality is deficiency of factor VIII. The severity of bleeding generally correlates with factor VIII level. Musculoskeletal bleeding namely, hemarthrosis is considered the main presenting manifestation of hemophilia A. ${ }^{1}$

The main treatment for those patients is factor VIII replacement therapy, either on demand or on prophylactic basis. A major challenge of such therapy, especially in the severe forms of hemophilia A, is the development of neutralizing antibodies (inhibitors), which makes therapy ineffective. Bleeding episodes in patients with inhibitors do not respond to FVIII concentrates, and can result in progressive functional disability and even death if not managed appropriately. In addition, such complication leads to marked increase in the cost of treatment that is estimated to be $4-5$ folds the cost of treatment of patients without inhibitors. ${ }^{2}$
Among those with hemophilia A, 25-30\% with severe form who are previously untreated (PUP) will develop inhibitors. Inhibitor formation occurs early after initiation of replacement therapy. Most studies report inhibitors development after less than 50 cumulative treatment exposure days. Positive family history of inhibitors, race, and underlying mutation resulting in hemophilia are important genetic risk factors for inhibitors development. ${ }^{3}$

Up to date literature assessing risk factors for inhibitors development in the Arab countries is lacking. This study was conducted to explore the incidence of inhibitors, and to identify non-genetic risk factors of their development in patients with severe hemophilia A. Defining environmental risk factors among Omani patient would facilitate developing a prevention model.

\section{Aim of the work}

The aim of the current study was to assess the prevalence of factor VIII inhibitors among Omani haemophiliac children, and relationship of their development with different risk factors. 


\section{Patients and methods}

This is a retrospective cross- sectional study that included all patients with hemophilia A registered in the main tertiary care hospitals in Oman. Data were collected using the Hospital Information System (HIS), and included patient's demographic data (Medical Registration Number, current age, age at diagnosis and at first treatment, date of the first documented positive inhibitor and data on non-genetic risk factors:
i. Mode of treatment (on demand versus prophylaxis)
ii. Type and dose of the concentrate used
iii. Time interval of exposure to factor VIII till inhibitor development,
iv. Previous surgeries or major bleeding events \pm blood transfusion

Missing data were collected through phone interviews whenever possible. Access to data base was restricted to those involved in the study. All information collected was treated with strict confidentiality. No identifiable information was entered in the study database, or released in any verbal or written communications. The study was approved by the medical research and ethics committee, College of Medicine and Health Sciences, Sultan Qaboos University.

Collected data were initially fed into an excel spread sheet for statistical distribution, and then a statistical Package (SPSS) was used for data analysis.

\section{Results}

Out of 156 hemophilia cases registered in Oman, data were available for 78 patients who were included in the current analysis. Inhibitors were reported by Bethesda assay which was positive in 24 patients (out of 70 patients for whom data were documented), giving a prevalence rate of $\sim 35 \%$. The age of inhibitor development ranged between 16months and 21years $(109.4+79.9$ months $)$. Most of patients with positive inhibitors had low titer $<5$ Bethesda units $(87 \%)$. Table 1 demonstrates that there was no significant relation between inhibitor development and baseline factor VIII level or age at first infusion. Table 2 \& Figure 1 highlight the significant correlation between factor VIII prophylactic dose and inhibitor development. Other presumed risk factors were not significantly different between inhibitor positive/ inhibitor negative hemophiliac patients.

Table I Risk factors for development of inhibitors

\begin{tabular}{|c|c|c|c|c|c|c|}
\hline & $\begin{array}{l}\text { Number of data } \\
\text { available }\end{array}$ & $\begin{array}{l}\text { Inhibitor } \\
\text { (+ve) }\end{array}$ & $\begin{array}{l}\text { Inhibitor } \\
\text { (-ve) }\end{array}$ & $\begin{array}{l}\text { Mann- } \\
\text { Whitney }\end{array}$ & Wilcoxon W & P-value \\
\hline Baseline factor VIII level (u/ml) & 56 & $0.0915(15)$ & $0.214(4 I)$ & $1.021 *$ & $-0.536 \#$ & 0.594 \\
\hline $\begin{array}{l}\text { Mean Age at first treatment } \\
\text { (Months) }\end{array}$ & 35 & $15.5(7)$ & I8.63 (28) & 80.5 & 108.5 & 0.479 \\
\hline
\end{tabular}

*Levene's test for Equality of Variances

\# $\mathrm{t}$ test for Equality of Means

Table 2 Relation between the presence of inhibitors and different risk factors

\begin{tabular}{|c|c|c|c|c|c|}
\hline & Inhibitor (+ve) & Inhibitor (-ve) & Data available & Pearson Chi -square & $P$ value \\
\hline \multicolumn{3}{|l|}{ Surgery } & 43 & 0.043 & 0.835 \\
\hline Yes & 7 & 14 & & & \\
\hline No & 8 & 14 & & & \\
\hline \multicolumn{3}{|l|}{ Bleeding } & 64 & 1.649 & 0.199 \\
\hline Yes & 22 & 39 & & & \\
\hline No & 0 & 3 & & & \\
\hline \multicolumn{3}{|l|}{ Transfusion } & 46 & 0.942 & 0.332 \\
\hline Yes & 4 & 10 & & & \\
\hline No & 14 & 18 & & & \\
\hline \multicolumn{3}{|l|}{ Prophylaxis dose } & 56 & 7.398 & 0.025 \\
\hline Up to $500 \mathrm{u}$ & 13 & 19 & & & \\
\hline $50 I-1000 u$ & I & 15 & & & \\
\hline$>1000 u$ & 1 & 7 & & & \\
\hline \multicolumn{3}{|c|}{ Family history of inhibitors } & 46 & 0.922 & 0.337 \\
\hline Yes & 9 & 9 & & & \\
\hline No & 10 & 18 & & & \\
\hline \multicolumn{3}{|c|}{ Type of factor replacement } & 48 & 12.687 & 0.002 \\
\hline Plasma derived VIII & 5 & 21 & & & \\
\hline Recombinant VIII & 3 & 12 & & & \\
\hline Factor VII & 6 & $\mathrm{I}$ & & & \\
\hline \multicolumn{3}{|c|}{ Type of factor VIII concentrates } & 41 & 0.004 & 0.952 \\
\hline Plasma derived & 5 & 21 & & & \\
\hline Recombinant & 3 & 12 & & & \\
\hline
\end{tabular}




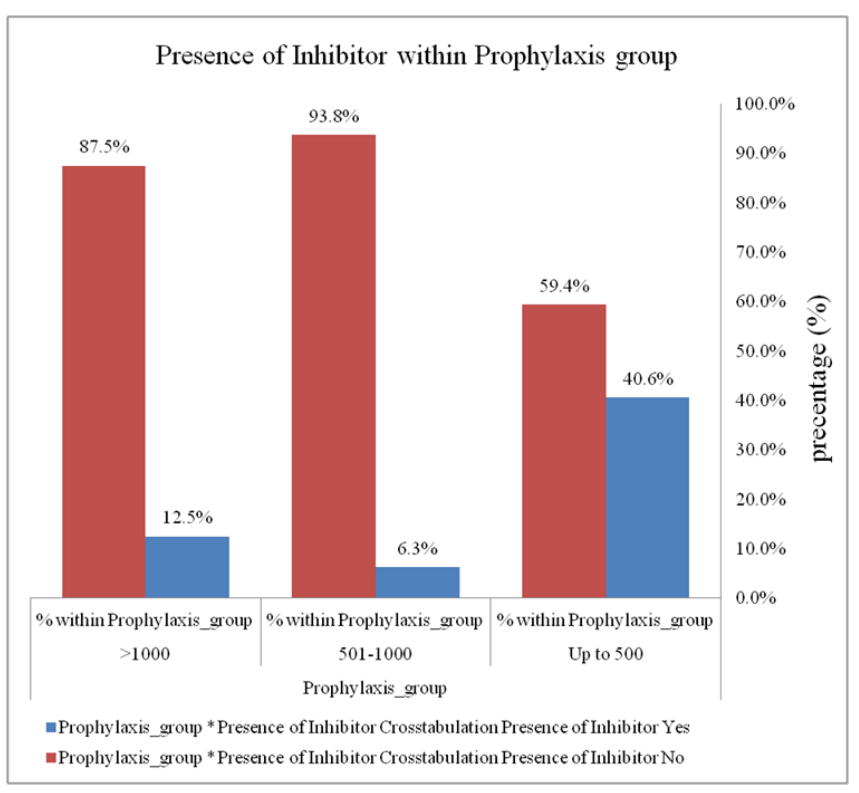

Figure I Correlation between dose of prophylactic factor VIII and the presence of inhibitors.

\section{Discussion}

Inhibitors development is a major complication in patients with hemophilia A in Oman. The reported prevalence of almost 35\% matches the international reported figures. Because of the seriousness of such complication and its impact on patient management, CDC in collaboration with hemophilia organizations and other partners were involved in the development of public health agenda to prevent the development of inhibitors, through national surveillance, epidemiological studies and promoting research that is directed towards solving the puzzles and filling the knowledge gap in this issue. $^{4}$

The prevalence of inhibitors in severe hemophilia A is estimated to be $25-30 \%$. Ethnicity plays a role in inhibitor development, reflecting the underlying prevalent factor VIII mutation, and polymorphism in different components of the immune system influencing reactivity to factor VIII concentrates. African- American and Latino patients are more prone to develop inhibitors than Caucasians. ${ }^{5}$

Reports from the Arab world regarding this concern are few. Owaida et al. ${ }^{6}$ reported a prevalence of factor VIII inhibitors of $29.3 \%$ among Saudi patients with hemophilia A. In contrast to our finding, they also reported that inhibitors were more frequent among patients receiving recombinant products. ${ }^{6}$

On the other hand, reports from Egypt revealed relatively low prevalence of inhibitors of 5\% with no impact on quality of life (QoL). ${ }^{7}$ In the current study, mean age at development of inhibitor was $(109.4+79.9)$ months, in agreement with previous evidence reporting an average age of 12 years for the development of inhibitors. ${ }^{8}$ The current study focused mainly on the non- genetic risk factors for development of inhibitors. Nevertheless, family history of inhibitors, that could be indirectly reflecting the impact of an underlying specific genetic mutation, had no significant impact on inhibitor development in our cohort. Such unexpected result may be attributed to the small sample size of the current study for whom such information was available (46 patients).

Non-genetic risk factors for development of inhibitors are regarded as "modifiable", thus, their identification and prevention can have a tremendous effect on individual patient management and future. Stimuli that alert the immune system at vulnerable time points for example microbial agents or vaccine, severe infections and tissue damage secondary to trauma, massive bleeding or surgical procedures are important danger conditions. ${ }^{9}$

In this respect, the current study did not show any significant difference between inhibitor positive and inhibitor negative patients in relation to the age at first factor VIII infusion, history of preceding surgical procedure, massive bleeding and/or blood transfusion. Although initial reports stated that exposure to factor VIII concentrates before 11 months of age increases the risk to develop inhibitors, more recent studies denied such correlation. ${ }^{10}$

In agreement with the current results, Chalmers et al, denoted that early exposure to factor VIII at different time points in the first year of life had no significant impact on the subsequent risk of inhibitor development. ${ }^{11}$ Data from the CANAL study confirmed the relation between intensity of treatment and risk to develop inhibitors. ${ }^{12}$ In the current study, history of surgical procedure and massive bleeding before development of inhibitors were used as indirect reflection of the intensity of factor VIII treatment in our cohort. This may explain the different results obtained as compared to CANAL study.

The current study did not show significant difference between inhibitor $+\mathrm{ve}$ and inhibitor $-\mathrm{ve}$ patients in relation to type of factor VIII concentrates (plasma-derived/ recombinant) ( $p$ 0.952). Data from previous studies gave conflicting results regarding this aspect. In agreement with our results, the CANAL study showed that the risk of inhibitor development was not substantially lower in patients receiving plasma- derived factor VIII concentrates compared to those treated with recombinant factors. ${ }^{12}$

Patients receiving relatively higher doses of factor VIII concentrates demonstrated significantly lower risk to develop inhibitors as compared to those receiving lower doses ( $\mathrm{p}$ 0.025). This might be explained by better control with less bleeding episodes, and hence less exposure to factor VIII concentrates at times of tissue damage. In agreement, Lorenzo et all and data from the CANAL study suggested that patients who are on factor VIII prophylaxis regimen have significantly lower risk to develop inhibitors than those who are treated on demand. ${ }^{12,13}$

\section{Conclusion and recommendations}

Inhibitors development is a major complication in patients with hemophilia A in Oman. The reported prevalence matches the international reported figures. Better control of bleeding episodes by using adequate prophylaxis, has positive impact on prevention of inhibitor development. A larger scale study that includes all the registered patients and their genetic mutation is needed.

\section{Acknowledgements}

We would like to thank our medical students Maryam Alkamzari and Noof Al-Belushi for helping in data collection. We would like also to thank our pediatric resident Adaweya Alajmi for participation in poster prepartaion of the current research. 


\section{Conflict of interest}

The authors declare that there is no conflict of interest.

\section{References}

1. Astermark J, Altisent C, Batorova A, et al. Non-genetic risk factors and the development of inhibitors in haemophilia: a comprehensive review and consensus report. Haemophilia. 2010;16(5):747-766.

2. Guh S, Grosse SD, McAlister S, et al. Healthcare expenditures for males with haemophilia and employer-sponsored insurance in the United States, 2008. Haemophilia. 2012;18(2):268-275.

3. Chambost H. Assessing risk factors: prevention of inhibitors in haemophilia. Haemophilia. 2010;16(Suppl 2):10-15.

4. Soucie JM, Miller CH, Kelly FM, et al. A public health approach to the prevention of inhibitors in hemophilia. Am JPrevMed. 2014;47(5):669-673.

5. Gunasekera D, Ettinger RA, Nakaya Fletcher S, et al. Factor VIII gene variants and inhibitor risk in African American hemophilia A patients. Blood. 2015;126(7):895-904.

6. Owaidah T, Momen AA, Alzahrani H, et al. The prevalence of factor VIII and IX inhibitors among Saudi patients with hemophilia: Results from the Saudi national hemophilia screening program. Medicine (Baltimore). 2017;96(2):e5456.
7. Tantawy AA, Mackensen SV, El-Laboudy MA, et al. Health-related quality of life in Egyptian children and adolescents with hemophilia A. Pediatr Hematol Oncol. 2011;28(3):222-229.

8. Donna M. DiMichele. Inhibitors in Haemophilia: A Primer. Treatment of hemophilia. 4th ed. World Federation of Haemophilia; 2008. p. 1-9.

9. Teresa Álvarez, Immaculada Soto, Jan Astermark. Non-genetic risk factors and their influence on the management of patients in the clinic. Eur J Haematol. 2015;94(Supp1 77):2-6.

10. Santagostino E, Mancuso ME, Rocino A, et al. Environmental risk factors for inhibitor development in children with haemophilia A: a case-control study. Br J Haematol. 2005;130(3):422-427.

11. Chalmers EA, Brown SA, Keeling D, et al. Early factor VIII exposure and subsequent inhibitor development in children with severe haemophilia A. Haemophilia. 2007;13(2):149-155.

12. Gouw SC, Van der Bom JG, Marijke van den Berg H. Treatment-related risk factors of inhibitor development in previously untreated patients with hemophilia A: the CANAL cohort study. Blood. 2007;109(11):4648-4654.

13. Lorenzo JI, López A, Altisent C, et al. Incidence of factor VIII inhibitors in severe haemophilia: the importance of patient age. $\mathrm{Br} J$ Haematol. 2001;113(3):600-603. 